\title{
Generalization of Montgomery identity via Taylor formula on time scales
}

\author{
Sumaiya Malik ${ }^{1}$ (D), Khuram Ali Khan ${ }^{1}$ (D), Ammara Nosheen ${ }^{1 *}$ (1) and Khalid Mahmood Awan ${ }^{1}$ (B)
}

\section{"Correspondence:}

hammaran@gmail.com

'Department of Mathematics,

University of Sargodha, Sargodha, Pakistan

\begin{abstract}
In the current paper, a generalized Montgomery identity is obtained with the help of Taylor's formula on time scales. The obtained identity is used to establish Ostrowski inequality, mid-point inequality, and trapezoid inequality. Moreover, the weighted versions of generalized Montgomery identity and respective Ostrowski inequality are also discussed. Special cases are obtained for different time scales to obtain new and existing results.
\end{abstract}

MSC: Primary 26D15; 26A33; 26A51; secondary 26E70; 39B62

Keywords: Montgomery identity; Ostrowski inequality; Trapezoid inequality; Time scales calculus

\section{Introduction}

An identity due to Montgomery is used to acquire various novel inequalities, for example, Ostrowski type inequality, trapezoid inequality, Mohajani inequality, Čebysěv and Grüss inequalities.

The Montgomery identity given by Pečaríc in [18] is expressed as follows:

Let $g:\left[c_{1}, d_{1}\right] \rightarrow \mathbb{R}$ and $g^{\prime}:\left[c_{1}, d_{1}\right] \rightarrow \mathbb{R}$ be integrable, then

$$
g(x)=\frac{1}{d_{1}-c_{1}} \int_{c_{1}}^{d_{1}} g(p) d p+\int_{c_{1}}^{d_{1}} R(x, p) g^{\prime}(p) d p
$$

where

$$
R(x, p)=\left\{\begin{array}{ll}
\frac{p-c_{1}}{d_{1}-c_{1}}, & c_{1} \leq p \leq x, \\
\frac{p-d_{1}}{d_{1}-c_{1}}, & x<p \leq d_{1}
\end{array}\right\}
$$

Pečarić [20] obtained the weighted form of Montgomery identity which states that, for any $x \in\left[c_{1}, d_{1}\right]$,

$$
g(x)=\int_{c_{1}}^{d_{1}} z(p) g(p) d p+\int_{c_{1}}^{d_{1}} R_{z}(x, p) g^{\prime}(p) d p,
$$

(c) The Author(s) 2022. This article is licensed under a Creative Commons Attribution 4.0 International License, which permits use, sharing, adaptation, distribution and reproduction in any medium or format, as long as you give appropriate credit to the original author(s) and the source, provide a link to the Creative Commons licence, and indicate if changes were made. The images or other third party material in this article are included in the article's Creative Commons licence, unless indicated otherwise in a credit line to the material. If material is not included in the article's Creative Commons licence and your intended use is not permitted by statutory regulation or exceeds the permitted use, you will need to obtain permission directly from the copyright holder. To view a copy of this licence, visit http://creativecommons.org/licenses/by/4.0/. 
where $g:\left[c_{1}, d_{1}\right] \rightarrow \mathbb{R}$ is differentiable on $\left[c_{1}, d_{1}\right], g^{\prime}:\left[c_{1}, d_{1}\right] \rightarrow \mathbb{R}$ is integrable on $\left[c_{1}, d_{1}\right]$, and $z:\left[c_{1}, d_{1}\right] \rightarrow[0, \infty\rangle$ is some normalized weight function, which satisfy $\int_{c_{1}}^{d_{1}} z(p) d p=$ 1 , and $Z(p)=\int_{c_{1}}^{p} z(x) d x$ for $p \in\left[c_{1}, d_{1}\right], Z(p)=0$ for $p<c_{1}$, and $Z(p)=1$ for $p>d_{1}$. The weighted Peano kernel is

$$
R_{z}(x, p)=\left\{\begin{array}{ll}
Z(p), & c_{1} \leq p \leq x \\
Z(p)-1, & x<p \leq d_{1}
\end{array}\right\} .
$$

The theory of time scales was firstly presented by S. Hilger in 1988. With the help of time scale theory, difference and differential equations are solved by a unified approach. The solutions are obtained for a real-valued functions on a closed subset $\mathbb{T}$ of $\mathbb{R}$ by extending the standard methods of calculus. Based on time scales theory $[7,9,10]$, further studies on integral inequalities on time scales are noted in literature. Bohner and Matthews [8] used time scale theory as a reference to obtain the time-scaled Montgomery identity and particular Ostrowski inequality.

Theorem $1\left(\left[8\right.\right.$, Lemma 3.1]) Let $c_{1}, d_{1}, s, p \in \mathbb{T}, c_{1}<d_{1}$, and $g:\left[c_{1}, d_{1}\right]_{\mathbb{T}}=\left[c_{1}, d_{1}\right] \cap \mathbb{T} \rightarrow \mathbb{R}$ be differentiable, then

$$
g(p)=\frac{1}{d_{1}-c_{1}} \int_{c_{1}}^{d_{1}} g^{\sigma}(s) \Delta s+\frac{1}{d_{1}-c_{1}} \int_{c_{1}}^{d_{1}} R(p, s) g^{\Delta}(s) \Delta s,
$$

where

$$
R(p, s)=\left\{\begin{array}{ll}
s-c_{1}, & c_{1} \leq s \leq p, \\
s-d_{1}, & p<s \leq d_{1}
\end{array}\right\} .
$$

The weighted Montgomery identity given in [21] on time scales is stated as follows.

Theorem 2 Let $c_{1}, d_{1}, s, p \in \mathbb{T}, c_{1}<d_{1}$, and $g:\left[c_{1}, d_{1}\right]_{\mathbb{T}}=\left[c_{1}, d_{1}\right] \cap \mathbb{T} \rightarrow \mathbb{R}$ be differentiable, then

$$
g(p)=\int_{c_{1}}^{d_{1}} z(s) g^{\sigma}(s) \Delta s+\int_{c_{1}}^{d_{1}} R_{z}(p, s) g^{\Delta}(s) \Delta s,
$$

where

$$
\begin{gathered}
R_{z}(p, s)=\left\{\begin{array}{ll}
Z(s), & c_{1} \leq s \leq p, \\
Z(s)-1, & p<s \leq d_{1}
\end{array}\right\}, \\
\text { and } z:\left[c_{1}, d_{1}\right]_{\mathbb{T}} \rightarrow[0, \infty), \int_{c_{1}}^{d_{1}} z(p) \Delta p=1, \\
Z(p)= \begin{cases}\int_{c_{1}}^{p} z(x) \Delta x, & p \in\left[c_{1}, d_{1}\right], \\
0, & p<c_{1}, \\
1, & p>d_{1}\end{cases}
\end{gathered}
$$

In this paper, an extension of Montgomery identity (3) is obtained by using the time scale versions of Taylor series which can be found in $[1,2,11]$. The obtained Montgomery identity $[3,14-17]$ is further used for time-scaled trapezoid and Ostrowski type inequalities 
$[5,6,12,19,22]$. Additionally, uncommon instances of Ostrowski inequality include a generalized mid-point inequality. Finally, the extension of (4) and the respective Ostrowski inequality is discussed.

\section{Preliminary results}

Some basic essentials regarding theory of time scales can be found in [7, 9, 10]. Few of which are given here: Generalized polynomials on time scales are the functions $u_{l}, v_{l}$ : $\mathbb{T}^{2} \rightarrow \mathbb{R}, l \in \mathbb{N}_{0}$ defined recursively as follows: $u_{0}(p, s)=v_{0}(p, s)=1, \forall p, s \in \mathbb{T}$ and for given $u_{l}, v_{l}$ with $l \in \mathbb{N}_{0}$,

$$
v_{l+1}(p, s)=\int_{s}^{p} v_{l}(\tau, s) \Delta \tau, \quad u_{l+1}(p, s)=\int_{s}^{p} u_{l}(\sigma(\tau), s) \Delta \tau .
$$

If $v_{l}^{\Delta}(p, s)$ presents each fixed $s \in \mathbb{T}$, the derivative for $v_{l+1}(p, s)$ with respect to $p$ is

$$
v_{l+1}^{\Delta}(p, s)=v_{l}(p, s), \quad u_{l+1}^{\Delta}(p, s)=u_{l}(\sigma(p), s) \quad \text { for } l \in \mathbb{N}_{0}, p \in \mathbb{T}^{k}
$$

where

$$
\mathbb{T}^{k}=\left\{\begin{array}{ll}
\mathbb{T}-(\rho(\sup \mathbb{T}), \sup \mathbb{T}] & \text { if } \sup \mathbb{T}<\infty \\
\mathbb{T} & \text { if } \sup \mathbb{T}=\infty
\end{array}\right\}
$$

Also

$$
v_{l}(p, s)=(-1)^{l} u_{l}(s, p)
$$

Taylor formula for random time scale $\mathbb{T}$ is stated below.

Theorem $3\left(\left[9\right.\right.$, Theorem 1.113]) Let $m \in \mathbb{N}, g$ be $m$ times differentiable on $\mathbb{T}^{k^{m}}$. Let $\alpha \in$ $\mathbb{T}^{k^{m-1}}, p \in \mathbb{T}$, then we have

$$
g(p)=\sum_{l=0}^{m-1} v_{l}(p, \alpha) g^{\Delta^{l}}(\alpha)+\int_{\alpha}^{\rho^{m-1}(p)} v_{m-1}(p, \sigma(\tau)) g^{\Delta^{m}}(\tau) \Delta \tau
$$

where $v_{l}: \mathbb{T}^{2} \rightarrow \mathbb{R}, l \in \mathbb{N}_{0}$ represents the generalized polynomial defined above.

In order to deal with double integrals on time sales, Basşak Karpuz [13, Lemma 1] proved the following result for exchange of integrals.

Lemma 1 Assume $s, p \in \mathbb{T}$ and $G \in C_{r d}(\mathbb{T} \times \mathbb{T}, \mathbb{R})$. Then

$$
\int_{s}^{p} \int_{\eta}^{p} G(\eta, \xi) \Delta \xi \Delta \eta=\int_{s}^{p} \int_{s}^{\sigma(\xi)} G(\eta, \xi) \Delta \eta \Delta \xi
$$

In a similar fashion, results obtained are shown below.

Lemma 2 Assume $s, p \in \mathbb{T}$ and $G \in C_{r d}(\mathbb{T} \times \mathbb{T}, \mathbb{R})$. Then

$$
\int_{s}^{p} \int_{s}^{\eta} G(\eta, \xi) \Delta \xi \Delta \eta=\int_{s}^{p} \int_{\sigma(\xi)}^{p} G(\eta, \xi) \Delta \eta \Delta \xi
$$


Proof Let

$$
g(p):=\int_{s}^{p} \int_{s}^{\eta} G(\eta, \xi) \Delta \xi \Delta \eta-\int_{s}^{p} \int_{\sigma(\xi)}^{p} G(\eta, \xi) \Delta \eta \Delta \xi
$$

for $p \in \mathbb{T}$. Then, by taking derivative and applying [9, Theorem 1.117], we have

$$
\begin{aligned}
g^{\Delta}(p) & :=\left(\int_{s}^{p} \int_{s}^{\eta} G(\eta, \xi) \Delta \xi \Delta \eta\right)^{\Delta}-\left(\int_{s}^{p} \int_{\sigma(\xi)}^{p} G(\eta, \xi) \Delta \eta \Delta \xi\right)^{\Delta} \\
& =\int_{s}^{p} f(p, \xi) \Delta \xi-\int_{s}^{p} \frac{\partial}{\partial p}\left(\int_{\sigma(\xi)}^{p} f(\eta, \xi) \Delta \eta\right) \Delta \xi-\int_{\sigma(p)}^{\sigma(p)} f(\eta, \xi) \Delta \eta \\
g^{\Delta}(p) & :=\int_{s}^{p} f(p, \xi) \Delta \xi-\int_{s}^{p} f(p, \xi) \Delta \xi=0,
\end{aligned}
$$

which proved the required result.

Remark 1 From [9, Theorem 1.109], it is straightforward that

$$
u_{m}\left(\rho^{l}(p), p\right)=0 \quad \forall m \in \mathbb{N}, 0 \leq l \leq m-1 .
$$

Lemma 3 The functions $u_{m}, m \in \mathbb{N}$ defined above satisfy, for all $p \in \mathbb{T}$,

$$
u_{m}\left(p, \rho^{l}(p)\right)=0 \quad \forall m \in \mathbb{N}, 0 \leq l \leq m-1 .
$$

Proof Here, the induction method is used to prove the result. For $l=0$,

$$
u_{m}\left(p, \rho^{0}(p)\right)=u_{m}(p, p)=0 .
$$

To conclude the induction, it will be sufficient that

$$
u_{m-1}\left(p, \rho^{l}(p)\right)=u_{m}\left(p, \rho^{l}(p)\right)=0, \quad 0 \leq l<m,
$$

implies that

$$
u_{m}\left(p, \rho^{l+1}(p)\right)=0 .
$$

If $\rho^{l}(p)$ is left-dense, then $\rho^{l+1}(p)=\rho^{l}(p)$ so that

$$
u_{m}\left(p, \rho^{l+1}(p)\right)=u_{m}\left(p, \rho^{l}(p)\right)=0 .
$$

If $\rho^{l}(p)$ is not left-dense, then it is left-scattered and $\sigma\left(\rho^{l+1}(p)\right)=\rho^{l}(p)$, therefore by [9, Theorem 1.16(iv)] we have

$$
\begin{aligned}
u_{m}\left(p, \rho^{l+1}(p)\right) & =u_{m}\left(p, \sigma\left(\rho^{l+1}(p)\right)\right)-\mu\left(\rho^{l+1}(p)\right) u_{m}^{\Delta}\left(p, \rho^{l+1}(p)\right) \\
& =u_{m}\left(p, \rho^{l}(p)\right)-\mu\left(\rho^{l+1}(p)\right) u_{m-1}\left(p, \sigma\left(\rho^{l+1}(p)\right)\right) \\
& =u_{m}\left(p, \rho^{l}(p)\right)-\mu\left(\rho^{l+1}(p)\right) u_{m-1}\left(p, \rho^{l+1}(p)\right)=0 .
\end{aligned}
$$

It proves our claim. 
The lemma shown below is helpful in proving the main result.

Lemma 4 The function $v_{l}$ for $p \in \mathbb{T}$ satisfies

$$
v_{m}\left(\rho^{l}(p), \sigma(p)\right)=0, \quad \forall m \in \mathbb{N}, 0 \leq l \leq m-2 .
$$

Proof By using Lemma 3, we can write $u_{m}\left(p, \rho^{l}(p)\right)=0 \forall m \in \mathbb{N}, 0 \leq l \leq m-1$. It is known that $v_{m}(p, s)=(-1)^{m} u_{m}(s, p), \forall m \in \mathbb{N}$. Thus we have

$$
v_{m}\left(\rho^{l}(p), p\right)=0, \quad \forall m \in \mathbb{N}, 0 \leq l \leq m-1 .
$$

By using [9, Theorem 1.16 (iv)],

$$
\begin{aligned}
& v_{m}\left(\rho^{l}(p), \sigma(p)\right)=v_{m}\left(\rho^{l}(p), p\right)+\mu(p) v_{m-1}\left(\rho^{l}(p), p\right) \\
& \quad \Rightarrow \quad v_{m}\left(\rho^{l}(p), \sigma(p)\right)=0, \quad \forall m \in \mathbb{N}, 0 \leq l \leq m-2 .
\end{aligned}
$$

\section{Generalization of Montgomery identity on time scales}

Theorem 4 Let $m \in \mathbb{N}, g$ be $m$ times differentiable on $\mathbb{T}^{k^{m}}$. Let $p \in \mathbb{T}$, then we have

$$
\begin{aligned}
g(p)= & \frac{1}{d_{1}-c_{1}} \int_{c_{1}}^{d_{1}} g^{\sigma}(s) \Delta s \\
& +\frac{1}{d_{1}-c_{1}} \sum_{l=0}^{m-2} g^{\Delta^{l+1}}\left(c_{1}\right)\left\{v_{l+1}\left(p, c_{1}\right)\left(p-c_{1}\right)-\int_{c_{1}}^{p} v_{l+1}\left(\sigma(s), c_{1}\right) \Delta s\right\} \\
& +\frac{1}{d_{1}-c_{1}} \sum_{l=0}^{m-2} g^{\Delta^{l+1}}\left(d_{1}\right)\left\{\int_{d_{1}}^{p} v_{l+1}\left(\sigma(s), d_{1}\right) \Delta s-v_{l+1}\left(p, d_{1}\right)\left(p-d_{1}\right)\right\} \\
& +\frac{1}{d_{1}-c_{1}} \int_{c_{1}}^{d_{1}} Q_{m}(p, \tau) g^{\Delta^{m}}(\tau) \Delta \tau,
\end{aligned}
$$

where

$$
Q_{m}(p, \tau)=\left[\begin{array}{c}
v_{m-1}(p, \sigma(\tau))\left(p-c_{1}\right)-\int_{\rho^{m-3}(\tau)}^{p} v_{m-1}(\sigma(s), \sigma(\tau)) \Delta s, \tau \in\left[c_{1}, p\right), \\
v_{m-1}(p, \sigma(\tau))\left(p-d_{1}\right)-\int_{\rho^{m-3}(\tau)}^{p} v_{m-1}(\sigma(s), \sigma(\tau)) \Delta s, \tau \in\left[p, d_{1}\right]
\end{array}\right]
$$

Proof Suppose that $g^{\Delta}$ is $m-1$ times differentiable, then by replacing $m$ with $m-1, g$ with $g^{\Delta}$, and $\alpha=c_{1}$ in (7), we have

$$
g^{\Delta}(p)=\sum_{l=0}^{m-2} v_{l}\left(p, c_{1}\right) g^{\Delta^{l+1}}\left(c_{1}\right)+\int_{c_{1}}^{\rho^{m-2}(p)} v_{m-2}(p, \sigma(\tau)) g^{\Delta^{m}}(\tau) \Delta \tau .
$$

Replace $c_{1}$ with $d_{1}$ in (11) to get

$$
g^{\Delta}(p)=\sum_{l=0}^{m-2} v_{l}\left(p, d_{1}\right) g^{\Delta^{l+1}}\left(d_{1}\right)+\int_{d_{1}}^{\rho^{m-2}(p)} v_{m-2}(p, \sigma(\tau)) g^{\Delta^{m}}(\tau) \Delta \tau .
$$


Malik et al. Journal of Inequalities and Applications

(2022) 2022:24

Page 6 of 17

We can rewrite (3) as

$$
\begin{aligned}
g(p)= & \frac{1}{d_{1}-c_{1}} \int_{c_{1}}^{d_{1}} g^{\sigma}(s) \Delta s+\frac{1}{d_{1}-c_{1}} \int_{c_{1}}^{p}\left(s-c_{1}\right) g^{\Delta}(s) \Delta s \\
& +\frac{1}{d_{1}-c_{1}} \int_{p}^{d_{1}}\left(s-d_{1}\right) g^{\Delta}(s) \Delta s .
\end{aligned}
$$

By using (11) and (12) in (13),

$$
\begin{aligned}
g(p)= & \frac{1}{d_{1}-c_{1}} \int_{c_{1}}^{d_{1}} g^{\sigma}(s) \Delta s \\
& +\frac{1}{d_{1}-c_{1}} \int_{c_{1}}^{p}\left(s-c_{1}\right) \sum_{l=0}^{m-2} v_{l}\left(s, c_{1}\right) g^{\Delta^{l+1}}\left(c_{1}\right) \Delta s \\
& +\frac{1}{d_{1}-c_{1}} \int_{p}^{d_{1}}\left(s-d_{1}\right) \sum_{l=0}^{m-2} v_{l}\left(s, d_{1}\right) g^{\Delta^{l+1}}\left(d_{1}\right) \Delta s \\
& +\frac{1}{d_{1}-c_{1}} \int_{c_{1}}^{p}\left(s-c_{1}\right) \int_{c_{1}}^{\rho^{m-2}(s)} v_{m-2}(s, \sigma(\tau)) g^{\Delta^{m}}(\tau) \Delta \tau \Delta s \\
& -\frac{1}{d_{1}-c_{1}} \int_{p}^{d_{1}}\left(s-d_{1}\right) \int_{\rho^{m-2}(s)}^{d_{1}} v_{m-2}(s, \sigma(\tau)) g^{\Delta^{m}}(\tau) \Delta \tau \Delta s .
\end{aligned}
$$

By making calculations for integral in (14),

$$
\begin{aligned}
\int_{c_{1}}^{p} & \left(s-c_{1}\right) \sum_{l=0}^{m-2} v_{l}\left(s, c_{1}\right) g^{\Delta^{l+1}}\left(c_{1}\right) \Delta s \\
= & \sum_{l=0}^{m-2} g^{\Delta^{l+1}}\left(c_{1}\right) \int_{c_{1}}^{p}\left(s-c_{1}\right) v_{l}\left(s, c_{1}\right) \Delta s \\
= & \sum_{l=0}^{m-2} g^{\Delta^{l+1}}\left(c_{1}\right) \int_{c_{1}}^{p} v_{l+1}^{\Delta}\left(s, c_{1}\right) v_{1}\left(s, c_{1}\right) \Delta s \\
= & \sum_{l=0}^{m-2} g^{\Delta^{l+1}}\left(c_{1}\right)\left\{v_{l+1}\left(p, c_{1}\right) v_{1}\left(p, c_{1}\right)-\int_{c_{1}}^{p} v_{l+1}\left(\sigma(s), c_{1}\right) \Delta s\right\} \\
= & \sum_{l=0}^{m-2} g^{\Delta^{l+1}}\left(c_{1}\right)\left\{v_{l+1}\left(p, c_{1}\right)\left(p-c_{1}\right)-\int_{c_{1}}^{p} v_{l+1}\left(\sigma(s), c_{1}\right) \Delta s\right\} .
\end{aligned}
$$

Similarly (15) gives

$$
\begin{aligned}
& \int_{p}^{d_{1}}\left(s-d_{1}\right) \sum_{l=0}^{m-2} v_{l}\left(s, d_{1}\right) g^{\Delta^{l+1}}\left(d_{1}\right) \Delta s \\
& \quad=\sum_{l=0}^{m-2} g^{\Delta^{l+1}}\left(d_{1}\right)\left\{\int_{d_{1}}^{p} v_{l+1}\left(\sigma(s), d_{1}\right) \Delta s-v_{l+1}\left(p, d_{1}\right)\left(p-d_{1}\right)\right\} .
\end{aligned}
$$


By using Lemma 2, integral in (16) takes the following form:

$$
\begin{aligned}
& \int_{c_{1}}^{p}\left(s-c_{1}\right) \int_{c_{1}}^{\rho^{m-2}(s)} v_{m-2}(s, \sigma(\tau)) g^{\Delta^{m}}(\tau) \Delta \tau \Delta s \\
& =\int_{c_{1}}^{p} g^{\Delta^{m}}(\tau) \int_{\rho^{m-3}(\tau)}^{p}\left(s-c_{1}\right) v_{m-2}(s, \sigma(\tau)) \Delta s \Delta \tau \\
& =\int_{c_{1}}^{p} g^{\Delta^{m}}(\tau) \int_{\rho^{m-3}(\tau)}^{p} v_{m-1}^{\Delta}(s, \sigma(\tau)) v_{1}\left(s, c_{1}\right) \Delta s \Delta \tau \\
& =\int_{c_{1}}^{p} g^{\Delta^{m}}(\tau)\left\{v_{m-1}(p, \sigma(\tau)) v_{1}\left(p, c_{1}\right)-v_{m-1}\left(\rho^{m-3}(\tau), \sigma(\tau)\right) v_{1}\left(\rho^{m-3}(\tau), c_{1}\right)\right\} \Delta \tau \\
& \quad-\int_{c_{1}}^{p} g^{\Delta^{m}}(\tau) \int_{\rho^{m-3}(\tau)}^{p} v_{m-1}(\sigma(s), \sigma(\tau)) \Delta s \Delta \tau .
\end{aligned}
$$

Lemma 4 implies $v_{m-1}\left(\rho^{m-3}(\tau), \sigma(\tau)\right)=0$.

$$
\begin{aligned}
& \Rightarrow \int_{c_{1}}^{p}\left(s-c_{1}\right) \int_{c_{1}}^{\rho^{m-2}(s)} v_{m-2}(s, \sigma(\tau)) g^{\Delta^{m}}(\tau) \Delta \tau \Delta s \\
& \quad=\int_{c_{1}}^{p} g^{\Delta^{m}}(\tau)\left\{v_{m-1}(p, \sigma(\tau))\left(p-c_{1}\right)-\int_{\rho^{m-3}(\tau)}^{p} v_{m-1}(\sigma(s), \sigma(\tau)) \Delta s\right\} \Delta \tau .
\end{aligned}
$$

Similarly, we have

$$
\begin{aligned}
& \int_{p}^{d_{1}}\left(s-d_{1}\right) \int_{\rho^{m-2}(s)}^{d_{1}} v_{m-2}(s, \sigma(\tau)) g^{\Delta^{m}}(\tau) \Delta \tau \Delta s \\
& \quad=\int_{d_{1}}^{p}\left(s-d_{1}\right) \int_{d_{1}}^{\rho^{m-2}(s)} v_{m-2}(s, \sigma(\tau)) g^{\Delta^{m}}(\tau) \Delta \tau \Delta s \\
& =\int_{d_{1}}^{p} g^{\Delta^{m}}(\tau)\left\{v_{m-1}(p, \sigma(\tau))\left(p-d_{1}\right)-\int_{\rho^{m-3}(\tau)}^{p} v_{m-1}(\sigma(s), \sigma(\tau)) \Delta s\right\} \Delta \tau \\
& =-\int_{p}^{d_{1}} g^{\Delta^{m}}(\tau)\left\{v_{m-1}(p, \sigma(\tau))\left(p-d_{1}\right)-\int_{\rho^{m-3}(\tau)}^{p} v_{m-1}(\sigma(s), \sigma(\tau)) \Delta s\right\} \Delta \tau
\end{aligned}
$$

Use (18)-(21) in (14)-(17) respectively to get the ideal outcome.

\section{Example 1}

- By using $\mathbb{T}=\mathbb{R}$ in (10), we get $[4$, Remark 1$]$.

- For $\mathbb{T}=\mathbb{Z},(10)$ transforms as

$$
\begin{aligned}
g(p)= & \frac{1}{d_{1}-c_{1}} \sum_{s=c_{1}+1}^{d_{1}} g(s) \\
& +\frac{1}{d_{1}-c_{1}} \sum_{l=1}^{m-1} \Delta^{l} g\left(c_{1}\right)\left\{\frac{\left(p-c_{1}\right)\left(p-c_{1}\right)^{(l)}}{(l) !}-\frac{\left(p+1-c_{1}\right)^{(l+1)}}{(l+1) !}\right\}
\end{aligned}
$$




$$
\begin{aligned}
& +\frac{1}{d_{1}-c_{1}} \sum_{l=1}^{m-1} \Delta^{l} g\left(d_{1}\right)\left\{\frac{\left(p+1-d_{1}\right)^{(l+1)}}{(l+1) !}-\frac{\left(p-d_{1}\right)\left(p-d_{1}\right)^{(l)}}{(l) !}\right\} \\
& +\frac{1}{d_{1}-c_{1}} \sum_{\tau=c_{1}}^{d_{1}-1} \Delta^{m} g(\tau) Q_{m}(p, \tau),
\end{aligned}
$$

where

$$
Q_{m}(p, \tau)=\left[\begin{array}{ll}
\frac{\left(p-c_{1}\right)(p-\tau-1)(m-1)}{(m-1) !}-\sum_{s=\tau-m+3}^{p-1} \frac{(s-\tau)^{(m-1)}}{(m-1) !}, & \tau \in\left[c_{1}, p\right) \\
\frac{\left(p-d_{1}\right)(p-\tau-1)(m-1)}{(m-1) !}-\sum_{s=\tau-m+3}^{p-1} \frac{(s-\tau)^{(m-1)}}{(m-1) !}, & \tau \in\left[p, d_{1}\right]
\end{array}\right]
$$

- For $\mathbb{T}=q^{\mathbb{Z}}, q>1,(10)$ takes the form

$$
\begin{aligned}
g(p)= & \frac{q-1}{d_{1}-c_{1}} \sum_{s=c_{1}}^{q^{-1} d_{1}} s f(q s) \\
& +\frac{1}{d_{1}-c_{1}} \sum_{l=1}^{m-1} \Delta^{l} g\left(c_{1}\right)\left\{\prod_{\nu=0}^{l-1} \frac{\left(p-q^{v} c_{1}\right)\left(p-c_{1}\right)}{\sum_{\mu=0}^{v} q^{\mu}}-q(q-1) \sum_{s=c_{1}}^{q^{-1} p} s \prod_{\nu=0}^{l-1} \frac{\left(s-q^{\nu-1} c_{1}\right)}{\sum_{\mu=0}^{v} q^{\mu}}\right\} \\
& +\frac{1}{d_{1}-c_{1}} \\
& \times \sum_{l=1}^{m-1} \Delta^{l} g\left(d_{1}\right)\left\{q(q-1) \sum_{s=d_{1}}^{q^{-1} p} \prod_{\nu=0}^{l-1} \frac{\left(s-q^{\nu-1} d_{1}\right)}{\sum_{\mu=0}^{v} q^{\mu}}-\prod_{\nu=0}^{l-1} \frac{\left(p-q^{v} d_{1}\right)\left(p-d_{1}\right)}{\sum_{\mu=0}^{v} q^{\mu}}\right\} \\
& +\frac{1}{d_{1}-c_{1}} \sum_{\tau=c_{1}}^{q^{-1} d_{1}} \Delta^{m} g(\tau) Q_{m}(p, \tau),
\end{aligned}
$$

where

$$
Q_{m}(p, \tau)=\left[\begin{array}{l}
\prod_{v=0}^{m-2} \frac{\left(p-q^{v+1} \tau\right)\left(p-c_{1}\right)}{\sum_{\mu=0}^{v} q^{\mu}}-q(q-1) \sum_{s=q^{3-m} \tau}^{q^{-1} p} s \prod_{\nu=0}^{m-2} \frac{\left(s-q^{\nu} \tau\right)}{\sum_{\mu=0}^{v} q^{\mu}}, \tau \in\left[c_{1}, p\right), \\
\prod_{v=0}^{m-2} \frac{\left(p-q^{v+1} \tau\right)\left(p-d_{1}\right)}{\sum_{\mu=0}^{\nu} q^{\mu}}-q(q-1) \sum_{s=q^{3-m_{\tau}}}^{q^{-1} p} s \prod_{v=0}^{m-2} \frac{\left(s-q^{\nu} \tau\right)}{\sum_{\mu=0}^{\nu} q^{\mu}}, \tau \in\left[p, d_{1}\right] .
\end{array}\right]
$$

Corollary 1 Using Theorem 4 and the corresponding conditions, we get the following generalized trapezoid inequality:

$$
\begin{aligned}
& \mid \frac{g\left(c_{1}\right)+g\left(d_{1}\right)}{2}-\frac{1}{d_{1}-c_{1}} \int_{c_{1}}^{d_{1}} g^{\sigma}(s) \Delta s \\
& \quad-\frac{1}{2\left(d_{1}-c_{1}\right)} \sum_{l=0}^{m-2} g^{\Delta^{l+1}}\left(c_{1}\right)\left\{v_{l+1}\left(d_{1}, c_{1}\right)\left(d_{1}-c_{1}\right)-\int_{c_{1}}^{d_{1}} v_{l+1}\left(\sigma(s), c_{1}\right) \Delta s\right\} \\
& \quad-\frac{1}{2\left(d_{1}-c_{1}\right)} \sum_{l=0}^{m-2} g^{\Delta^{l+1}}\left(d_{1}\right)\left\{-\int_{c_{1}}^{d_{1}} v_{l+1}\left(\sigma(s), d_{1}\right) \Delta s-v_{l+1}\left(c_{1}, d_{1}\right)\left(c_{1}-d_{1}\right)\right\} \mid \\
& \leq \frac{1}{2\left(d_{1}-c_{1}\right)}\left\|g^{\Delta^{m}}\right\|_{r}\left(\int_{c_{1}}^{d_{1}}\left|Q_{m}\left(c_{1}, \tau\right)+Q_{m}\left(d_{1}, \tau\right)\right|^{q} \Delta \tau\right)^{\frac{1}{q}} .
\end{aligned}
$$


Proof For the generalized trapezoid inequality, replace $p=c_{1}$ and $p=d_{1}$ in (10) to get the accompanying structures

$$
\begin{aligned}
g\left(c_{1}\right)= & \frac{1}{d_{1}-c_{1}} \int_{c_{1}}^{d_{1}} g^{\sigma}(s) \Delta s \\
& +\frac{1}{d_{1}-c_{1}} \sum_{l=0}^{m-2} g^{\Delta^{l+1}}\left(d_{1}\right)\left\{-\int_{c_{1}}^{d_{1}} v_{l+1}\left(\sigma(s), d_{1}\right) \Delta s-v_{l+1}\left(c_{1}, d_{1}\right)\left(c_{1}-d_{1}\right)\right\} \\
& +\frac{1}{d_{1}-c_{1}} \int_{c_{1}}^{d_{1}} Q_{m}\left(c_{1}, \tau\right) g^{\Delta^{m}}(\tau) \Delta \tau
\end{aligned}
$$

and

$$
\begin{aligned}
g\left(d_{1}\right)= & \frac{1}{d_{1}-c_{1}} \int_{c_{1}}^{d_{1}} g^{\sigma}(s) \Delta s \\
& +\frac{1}{d_{1}-c_{1}} \sum_{l=0}^{m-2} g^{\Delta^{l+1}}\left(c_{1}\right)\left\{v_{l+1}\left(d_{1}, c_{1}\right)\left(d_{1}-c_{1}\right)-\int_{c_{1}}^{d_{1}} v_{l+1}\left(\sigma(s), c_{1}\right) \Delta s\right\} \\
& +\frac{1}{d_{1}-c_{1}} \int_{c_{1}}^{d_{1}} Q_{m}\left(d_{1}, \tau\right) g^{\Delta^{m}}(\tau) \Delta \tau .
\end{aligned}
$$

Add (23) and (24) and divide the resultant by 2 to get

$$
\begin{aligned}
& \frac{g\left(c_{1}\right)+g\left(d_{1}\right)}{2}-\frac{1}{d_{1}-c_{1}} \int_{c_{1}}^{d_{1}} g^{\sigma}(s) \Delta s \\
& \quad-\frac{1}{2\left(d_{1}-c_{1}\right)} \sum_{l=0}^{m-2} g^{\Delta^{l+1}}\left(c_{1}\right)\left\{v_{l+1}\left(d_{1}, c_{1}\right)\left(d_{1}-c_{1}\right)-\int_{c_{1}}^{d_{1}} v_{l+1}\left(\sigma(s), c_{1}\right) \Delta s\right\} \\
& \quad-\frac{1}{2\left(d_{1}-c_{1}\right)} \sum_{l=0}^{m-2} g^{\Delta^{l+1}}\left(d_{1}\right)\left\{-\int_{c_{1}}^{d_{1}} v_{l+1}\left(\sigma(s), d_{1}\right) \Delta s-v_{l+1}\left(c_{1}, d_{1}\right)\left(c_{1}-d_{1}\right)\right\} \\
& =\frac{1}{2\left(d_{1}-c_{1}\right)} \int_{c_{1}}^{d_{1}}\left[Q_{m}\left(c_{1}, \tau\right)+Q_{m}\left(d_{1}, \tau\right)\right] g^{\Delta^{m}}(\tau) \Delta \tau
\end{aligned}
$$

By using Hölder's inequality on (27), we get

$$
\begin{aligned}
& \mid \frac{g\left(c_{1}\right)+g\left(d_{1}\right)}{2}-\frac{1}{d_{1}-c_{1}} \int_{c_{1}}^{d_{1}} g^{\sigma}(s) \Delta s \\
& \quad-\frac{1}{2\left(d_{1}-c_{1}\right)} \sum_{l=0}^{m-2} g^{\Delta^{l+1}}\left(c_{1}\right)\left\{v_{l+1}\left(d_{1}, c_{1}\right)\left(d_{1}-c_{1}\right)-\int_{c_{1}}^{d_{1}} v_{l+1}\left(\sigma(s), c_{1}\right) \Delta s\right\} \\
& \quad-\frac{1}{2\left(d_{1}-c_{1}\right)} \sum_{l=0}^{m-2} g^{\Delta^{l+1}}\left(d_{1}\right)\left\{-\int_{c_{1}}^{d_{1}} v_{l+1}\left(\sigma(s), d_{1}\right) \Delta s-v_{l+1}\left(c_{1}, d_{1}\right)\left(c_{1}-d_{1}\right)\right\} \mid \\
& \leq \frac{1}{2\left(d_{1}-c_{1}\right)}\left\|g^{\Delta^{m}}\right\|_{r}\left(\int_{c_{1}}^{d_{1}}\left|Q_{m}\left(c_{1}, \tau\right)+Q_{m}\left(d_{1}, \tau\right)\right|^{q} \Delta \tau\right)^{\frac{1}{q}},
\end{aligned}
$$


which is the required trapezoid inequality, where

$$
\begin{aligned}
& Q_{m}\left(c_{1}, \tau\right)+Q_{m}\left(d_{1}, \tau\right) \\
& =-2\left[-\int_{c_{1}}^{\rho^{m-3}(\tau)} v_{m-1}(\sigma(s), \sigma(\tau)) \Delta s+\int_{\rho^{m-3}(\tau)}^{d_{1}} v_{m-1}(\sigma(s), \sigma(\tau)) \Delta s\right] \\
& \quad+v_{m-1}\left(c_{1}, \sigma(\tau)\right)\left(c_{1}-d_{1}\right)+v_{m-1}\left(d_{1}, \sigma(\tau)\right)\left(d_{1}-c_{1}\right) .
\end{aligned}
$$

Remark 2 If $m=2$ and $q=1$ in Corollary 1, (22) takes the form

$$
\begin{aligned}
& \mid \frac{g\left(c_{1}\right)+g\left(d_{1}\right)}{2}-\frac{1}{d_{1}-c_{1}} \int_{c_{1}}^{d_{1}} g^{\sigma}(s) \Delta s \\
& \quad-\frac{1}{2\left(d_{1}-c_{1}\right)} g^{\Delta}\left(c_{1}\right)\left\{\left(d_{1}-c_{1}\right)^{2}-\int_{c_{1}}^{d_{1}}\left(\sigma(s)-c_{1}\right) \Delta s\right\} \\
& \quad-\frac{1}{2\left(d_{1}-c_{1}\right)} g^{\Delta}\left(d_{1}\right)\left\{-\int_{c_{1}}^{d_{1}}\left(\sigma(s)-d_{1}\right) \Delta s-\left(c_{1}-d_{1}\right)^{2}\right\} \mid \\
& \leq \frac{1}{2\left(d_{1}-c_{1}\right)}\left\|g^{\Delta^{2}}\right\|_{\infty} \int_{c_{1}}^{d_{1}}\left|Q_{2}\left(c_{1}, \tau\right)+Q_{2}\left(d_{1}, \tau\right)\right| \Delta \tau,
\end{aligned}
$$

where

$$
\begin{aligned}
& Q_{2}\left(c_{1}, \tau\right)+Q_{2}\left(d_{1}, \tau\right) \\
& =-2\left[-\int_{c_{1}}^{\rho^{-1}(\tau)} v_{1}(\sigma(s), \sigma(\tau)) \Delta s+\int_{\rho^{-1}(\tau)}^{d_{1}} v_{1}(\sigma(s), \sigma(\tau)) \Delta s\right] \\
& \quad+v_{1}\left(c_{1}, \sigma(\tau)\right)\left(c_{1}-d_{1}\right)+v_{1}\left(d_{1}, \sigma(\tau)\right)\left(d_{1}-c_{1}\right) .
\end{aligned}
$$

Remark 3 By using $\mathbb{T}=\mathbb{R}$ in (22), we get $[4$, Remark 3$]$.

\subsection{Ostrowski type inequality}

Theorem 5 Considering all taken assumptions of Theorem 4 hold, suppose that $(r, q)$ is a pair of conjugate exponents, that is, $1 \leq r, q<\infty, \frac{1}{r}+\frac{1}{q}=1$. Then we have

$$
\begin{aligned}
\mid g(p) & -\frac{1}{d_{1}-c_{1}} \int_{c_{1}}^{d_{1}} g^{\sigma}(s) \Delta s \\
& -\frac{1}{d_{1}-c_{1}} \sum_{l=0}^{m-2} g^{\Delta^{l+1}}\left(c_{1}\right)\left\{v_{l+1}\left(p, c_{1}\right)\left(p-c_{1}\right)-\int_{c_{1}}^{p} v_{l+1}\left(\sigma(s), c_{1}\right) \Delta s\right\} \\
& -\frac{1}{d_{1}-c_{1}} \sum_{l=0}^{m-2} g^{\Delta^{l+1}}\left(d_{1}\right)\left\{\int_{d_{1}}^{p} v_{l+1}\left(\sigma(s), d_{1}\right) \Delta s-v_{l+1}\left(p, d_{1}\right)\left(p-d_{1}\right)\right\} \mid \\
\leq & \frac{1}{d_{1}-c_{1}}\left\|g^{\Delta^{m}}\right\|\left(\int_{c_{1}}^{d_{1}}\left|Q_{m}(p, \tau)\right|^{q} \Delta \tau\right)^{\frac{1}{q}} .
\end{aligned}
$$

The constant $\left(\int_{c_{1}}^{d_{1}}\left|Q_{m}(p, \tau)\right|^{q} \Delta \tau\right)^{\frac{1}{q}}$ is sharp for $1<r \leq \infty$ and the best possible for $r=1$. 
Proof Employing identity (10) and Hölder's inequality, the following is obtained:

$$
\begin{aligned}
\mid g(p) & -\frac{1}{d_{1}-c_{1}} \int_{c_{1}}^{d_{1}} g^{\sigma}(s) \Delta s \\
& -\frac{1}{d_{1}-c_{1}} \sum_{l=0}^{m-2} g^{\Delta^{l+1}}\left(c_{1}\right)\left\{v_{l+1}\left(p, c_{1}\right)\left(p-c_{1}\right)-\int_{c_{1}}^{p} v_{l+1}\left(\sigma(s), c_{1}\right) \Delta s\right\} \\
& -\frac{1}{d_{1}-c_{1}} \sum_{l=0}^{m-2} g^{\Delta^{l+1}}\left(d_{1}\right)\left\{\int_{d_{1}}^{p} v_{l+1}\left(\sigma(s), d_{1}\right) \Delta s-v_{l+1}\left(p, d_{1}\right)\left(p-d_{1}\right)\right\} \mid \\
\leq & \frac{1}{d_{1}-c_{1}}\left\|g^{\Delta^{m}}\right\|\left(\int_{c_{1}}^{d_{1}}\left|Q_{m}(p, \tau)\right|^{q} \Delta \tau\right)^{\frac{1}{q}} .
\end{aligned}
$$

Denote $D_{1}(\tau)=Q_{m}(p, \tau)$. To verify the sharpness of the constant $\left(\int_{c_{1}}^{d_{1}}\left|D_{1}(\tau)\right|^{q} \Delta \tau\right)^{\frac{1}{q}}$, a function $g$ is constructed for which the correspondence in (28) is obtained.

For $1<r<\infty$, take $g$ with the end goal which states that

$$
g^{\Delta^{m}}(\tau)=\operatorname{sgn} D_{1}(\tau) \cdot\left|D_{1}(\tau)\right|^{\frac{1}{r-1}}
$$

For $r=\infty$, take

$$
g^{\Delta^{m}}(\tau)=\operatorname{sgn} D_{1}(\tau)
$$

For $r=1$, it will be proved that

$$
\left|\int_{c_{1}}^{d_{1}} D_{1}(\tau) g^{\Delta^{m}}(\tau) \Delta \tau\right| \leq \max _{\tau \in\left[c_{1}, d_{1}\right]_{\mathbb{T}}}\left|D_{1}(\tau)\right|\left(\int_{c_{1}}^{d_{1}}\left|g^{\Delta^{m}}(\tau)\right| \Delta \tau\right)
$$

is the optimal inequality. Suppose that $\left|D_{1}(\tau)\right|$ is maximum for $\tau_{0} \in\left[c_{1}, d_{1}\right]_{\mathbb{T}}$. First assume that $D_{1}\left(\tau_{0}\right)>0$ and for $\epsilon$ such that $0<\epsilon<d_{1}-\tau_{0}$; define $g_{\epsilon}(\cdot)$ by

$$
g_{\epsilon}(\tau)=\left\{\begin{array}{ll}
0, & c_{1} \leq \tau<\tau_{0}, \\
\frac{1}{\epsilon} v_{m}\left(\tau, \tau_{0}\right), & \tau_{0} \leq \tau<\tau_{0}+\epsilon, \\
\frac{1}{m} v_{m-1}\left(\tau, \tau_{0}\right), & \tau_{0}+\epsilon \leq \tau \leq d_{1} .
\end{array}\right\}
$$

For $\tau_{0} \leq \tau \leq \tau_{0}+\epsilon$, the expression for derivatives is

$$
\begin{aligned}
& g_{\epsilon}^{\prime}(\tau)=\frac{1}{\epsilon} v_{m}^{\Delta}\left(\tau, \tau_{0}\right)=\frac{1}{\epsilon} v_{m-1}\left(\tau, \tau_{0}\right), \\
& g_{\epsilon}^{\prime \prime}(\tau)=\frac{1}{\epsilon} v_{m-1}^{\Delta}\left(\tau, \tau_{0}\right)=\frac{1}{\epsilon} v_{m-2}\left(\tau, \tau_{0}\right) .
\end{aligned}
$$

Similarly, for $m$ - th derivative,

$$
\begin{aligned}
g_{\epsilon}^{\Delta^{m}}(\tau) & =\frac{1}{\epsilon} v_{m-m}\left(\tau, \tau_{0}\right)=\frac{1}{\epsilon} v_{0}\left(\tau, \tau_{0}\right) \\
& =\frac{1}{\epsilon} \quad \because v_{0}=1 .
\end{aligned}
$$


For $\tau_{0}+\epsilon \leq \tau \leq d_{1}$,

$$
\begin{aligned}
& g_{\epsilon}^{\prime}(\tau)=\frac{1}{m} v_{m-1}^{\Delta}\left(\tau, \tau_{0}\right)=\frac{1}{m} v_{m-2}\left(\tau, \tau_{0}\right), \\
& g_{\epsilon}^{\prime \prime}(\tau)=\frac{1}{m} v_{m-2}^{\Delta}\left(\tau, \tau_{0}\right)=\frac{1}{m} v_{m-3}\left(\tau, \tau_{0}\right)
\end{aligned}
$$

and

$$
g_{\epsilon}^{\Delta^{m}}(\tau)=\frac{1}{m} v_{m-m}^{\Delta}\left(\tau, \tau_{0}\right)=\frac{1}{m} v_{0}^{\Delta}=0 .
$$

For $\epsilon$ small enough,

$$
\left|\int_{c_{1}}^{d_{1}} D_{1}(\tau) g^{\Delta^{m}}(\tau) \Delta \tau\right|=\left|\int_{\tau_{0}}^{\tau_{0}+\epsilon} D_{1}(\tau) \frac{1}{\epsilon} \Delta \tau\right|=\frac{1}{\epsilon} \int_{\tau_{0}}^{\tau_{0}+\epsilon} D_{1}(\tau) \Delta \tau .
$$

(30) gives

$$
\frac{1}{\epsilon} \int_{\tau_{0}}^{\tau_{0}+\epsilon} D_{1}(\tau) \Delta \tau \leq D_{1}\left(\tau_{0}\right) \int_{\tau_{0}}^{\tau_{0}+\epsilon} \frac{1}{\epsilon} \Delta \tau=D_{1}\left(\tau_{0}\right) .
$$

Since

$$
\lim _{\epsilon \rightarrow 0} \frac{1}{\epsilon} \int_{\tau_{0}}^{\tau_{0}+\epsilon} D_{1}(\tau) \Delta \tau=D_{1}\left(\tau_{0}\right)
$$

Hence we have proved that equation (30) is an optimal inequality. For $D_{1}\left(\tau_{0}\right)<0$, we take

$$
g_{\epsilon}(\tau)=\left\{\begin{array}{ll}
\frac{1}{m} v_{m-1}\left(\tau, \tau_{0}+\epsilon\right), & c_{1} \leq \tau \leq \tau_{0}, \\
\frac{-1}{\epsilon} v_{m}\left(\tau, \tau_{0}+\epsilon\right), & \tau_{0} \leq \tau \leq \tau_{0}+\epsilon, \\
0, & \tau_{0}+\epsilon \leq \tau \leq d_{1} .
\end{array}\right\}
$$

To obtain a solution of the above function when $D_{1}\left(\tau_{0}\right)<0$, a similar method can be used as for $D_{1}\left(\tau_{0}\right)>0$.

Corollary 2 Considering taken conditions for Theorem 5 hold, for $r=1$, we have

$$
\begin{aligned}
\mid g(p) & -\frac{1}{d_{1}-c_{1}} \int_{c_{1}}^{d_{1}} g^{\sigma}(s) \Delta s \\
& -\frac{1}{d_{1}-c_{1}} \sum_{l=0}^{m-2} g^{\Delta^{l+1}}\left(c_{1}\right)\left\{v_{l+1}\left(p, c_{1}\right)\left(p-c_{1}\right)-\int_{c_{1}}^{p} v_{l+1}\left(\sigma(s), c_{1}\right) \Delta s\right\} \\
& -\frac{1}{d_{1}-c_{1}} \sum_{l=0}^{m-2} g^{\Delta^{l+1}}\left(d_{1}\right)\left\{\int_{d_{1}}^{p} v_{l+1}\left(\sigma(s), d_{1}\right) \Delta s-v_{l+1}\left(p, d_{1}\right)\left(p-d_{1}\right)\right\} \mid \\
\leq & \frac{1}{d_{1}-c_{1}}\left\|g^{\Delta^{m}}\right\| \max \left\{\left|v_{m-1}\left(p, \sigma\left(c_{1}\right)\right)\left(p-c_{1}\right)-\int_{\rho^{m-3}\left(c_{1}\right)}^{p} v_{m-1}\left(\sigma(s), \sigma\left(c_{1}\right)\right) \Delta s\right|,\right. \\
& \left.\left|v_{m-1}\left(p, \sigma\left(d_{1}\right)\right)\left(p-d_{1}\right)-\int_{\rho^{m-3}\left(d_{1}\right)}^{p} v_{m-1}\left(\sigma(s), \sigma\left(d_{1}\right)\right) \Delta s\right|\right\} .
\end{aligned}
$$


Malik et al. Journal of Inequalities and Applications

(2022) $2022: 24$

Page 13 of 17

Proof By using (10),

$$
\begin{aligned}
& \int_{c_{1}}^{d_{1}}\left|Q_{m}(p, \tau)\right|^{q} \Delta \tau \\
& =\int_{c_{1}}^{p}\left|Q_{m}(p, \tau)\right|^{q} \Delta \tau+\int_{p}^{d_{1}}\left|Q_{m}(p, \tau)\right|^{q} \Delta \tau \\
& =\int_{c_{1}}^{p}\left|v_{m-1}(p, \sigma(\tau))\left(p-c_{1}\right)-\int_{\rho^{m-3}(\tau)}^{p} v_{m-1}(\sigma(s), \sigma(\tau)) \Delta s\right|^{q} \Delta \tau \\
& \quad+\int_{p}^{d_{1}}\left|v_{m-1}(p, \sigma(\tau))\left(p-d_{1}\right)-\int_{\rho^{m-3}(\tau)}^{p} v_{m-1}(\sigma(s), \sigma(\tau)) \Delta s\right|^{q} \Delta \tau,
\end{aligned}
$$

$r=1 \Rightarrow q=\infty$, and we have

$$
\begin{aligned}
& \sup _{\tau \in\left[c_{1}, d_{1}\right]}\left|Q_{m}(p, \tau)\right| \\
& =\max \left\{\sup _{\tau \in\left[c_{1}, p\right]}\left|v_{m-1}(p, \sigma(\tau))\left(p-c_{1}\right)-\int_{\rho^{m-3}(\tau)}^{p} v_{m-1}(\sigma(s), \sigma(\tau)) \Delta s\right|,\right. \\
& \left.\quad \sup _{\tau \in\left[p, d_{1}\right]}\left|v_{m-1}(p, \sigma(\tau))\left(p-d_{1}\right)-\int_{\rho^{m-3}(\tau)}^{p} v_{m-1}(\sigma(s), \sigma(\tau)) \Delta s\right|\right\} \\
& =\max \left\{\left|v_{m-1}\left(p, \sigma\left(c_{1}\right)\right)\left(p-c_{1}\right)-\int_{\rho^{m-3}\left(c_{1}\right)}^{p} v_{m-1}\left(\sigma(s), \sigma\left(c_{1}\right)\right) \Delta s\right|\right. \\
& \left.\left|v_{m-1}\left(p, \sigma\left(d_{1}\right)\right)\left(p-d_{1}\right)-\int_{\rho^{m-3}\left(d_{1}\right)}^{p} v_{m-1}\left(\sigma(s), \sigma\left(d_{1}\right)\right) \Delta s\right|\right\} .
\end{aligned}
$$

By using the above expression in (28), we get (31).

Remark 4 Choose $m=2$ in Corollary 2. In this case (31) takes the form

$$
\begin{aligned}
\mid g(p) & -\frac{1}{d_{1}-c_{1}} \int_{c_{1}}^{d_{1}} g^{\sigma}(s) \Delta s-\frac{1}{d_{1}-c_{1}} g^{\Delta}\left(c_{1}\right)\left\{\left(p-c_{1}\right)^{2}-\int_{c_{1}}^{p}\left(\sigma(s)-c_{1}\right) \Delta s\right\} \\
& -\frac{1}{d_{1}-c_{1}} g^{\Delta}\left(d_{1}\right)\left\{\int_{d_{1}}^{p}\left(\sigma(s)-d_{1}\right) \Delta s-\left(p-d_{1}\right)^{2}\right\} \mid \\
\leq & \frac{1}{d_{1}-c_{1}}\left\|g^{\Delta^{2}}\right\| \max \left\{\left|\left(p-\sigma\left(c_{1}\right)\right)\left(p-c_{1}\right)-\int_{\rho^{-1}\left(c_{1}\right)}^{p}\left(\sigma(s)-\sigma\left(c_{1}\right)\right) \Delta s\right|,\right. \\
& \left.\left|\left(p-\sigma\left(d_{1}\right)\right)\left(p-d_{1}\right)-\int_{\rho^{-1}\left(d_{1}\right)}^{p}\left(\sigma(s)-\sigma\left(d_{1}\right)\right) \Delta s\right|\right\} .
\end{aligned}
$$

Remark 5 Use $p=\frac{c_{1}+d_{1}}{2}$ in Theorem 5. In this case (28) becomes the following generalzed midpoint inequality:

$$
\begin{aligned}
& \mid g\left(\frac{c_{1}+d_{1}}{2}\right)-\frac{1}{d_{1}-c_{1}} \int_{c_{1}}^{d_{1}} g^{\sigma}(s) \Delta s \\
& \quad-\frac{1}{d_{1}-c_{1}} \sum_{l=0}^{m-2} g^{\Delta^{l+1}}\left(c_{1}\right)\left\{v_{l+1}\left(\frac{c_{1}+d_{1}}{2}, c_{1}\right)\left(\frac{d_{1}-c_{1}}{2}\right)-\int_{c_{1}}^{\frac{c_{1}+d_{1}}{2}} v_{l+1}\left(\sigma(s), c_{1}\right) \Delta s\right\}
\end{aligned}
$$




$$
\begin{aligned}
& -\frac{1}{d_{1}-c_{1}} \sum_{k=0}^{m-2} g^{\Delta^{l+1}}\left(d_{1}\right)\left\{\int_{d_{1}}^{\frac{c_{1}+d_{1}}{2}} v_{l+1}\left(\sigma(s), d_{1}\right) \Delta s-v_{l+1}\left(\frac{c_{1}+d_{1}}{2}, d_{1}\right)\left(\frac{c_{1}-d_{1}}{2}\right)\right\} \mid \\
\leq & \frac{1}{d_{1}-c_{1}}\left\|g^{\Delta^{m}}\right\|_{r}\left(\int_{c_{1}}^{d_{1}}\left|Q_{m}\left(\frac{c_{1}+d_{1}}{2}, \tau\right)\right|^{q} \Delta \tau\right)^{\frac{1}{q}} .
\end{aligned}
$$

Remark 6 By using $\mathbb{T}=\mathbb{R}$ in Sect. 3.1, we get [4, Corollary 1, Remark 2, Remark 3].

\section{Weighted Montgomery identity}

Theorem 6 Let $m \in \mathbb{N}$ and $g$ be $m$ times differentiable on $\mathbb{T}^{k^{m}}$. Let $p \in \mathbb{T}$ and $z:\left[c_{1}, d_{1}\right]_{\mathbb{T}} \rightarrow$ $[0, \infty)$ be some probability density function, then we have

$$
\begin{aligned}
g(p)= & \int_{c_{1}}^{d_{1}} z(s) g^{\sigma}(s) \Delta s \\
& +\sum_{l=0}^{m-2} g^{\Delta^{l+1}}\left(c_{1}\right) \int_{c_{1}}^{p} z(s)\left\{v_{l+1}\left(p, c_{1}\right)-v_{l+1}\left(\sigma(s), c_{1}\right)\right\} \Delta s \\
& +\sum_{l=0}^{m-2} g^{\Delta^{l+1}}\left(d_{1}\right)\left[\int_{d_{1}}^{p} z(s)\left\{v_{l+1}\left(\sigma(s), d_{1}\right)-v_{l+1}\left(p, d_{1}\right)\right\} \Delta s\right] \\
& +\int_{c_{1}}^{d_{1}} Q_{z, m}(p, \tau) g^{\Delta^{m}}(\tau) \Delta \tau,
\end{aligned}
$$

where

$$
\begin{aligned}
& Q_{z, m}(p, \tau) \\
& \quad=\left[\begin{array}{l}
v_{m-1}(p, \sigma(\tau)) Z(p)-\int_{\rho^{m-3}(\tau)}^{p} v_{m-1}(\sigma(s), \sigma(\tau)) Z^{\Delta}(s) \Delta s, \quad \tau \in\left[c_{1}, p\right), \\
v_{m-1}(p, \sigma(\tau))(1-Z(p))+\int_{\rho^{m-3}(\tau)}^{p} v_{m-1}(\sigma(s), \sigma(\tau)) Z^{\Delta}(s) \Delta s, \quad \tau \in\left[p, d_{1}\right],
\end{array}\right]
\end{aligned}
$$

and the term $Z(p)$ involved in kernel is defined in (6).

Proof Since $g^{\Delta}$ is $m-1$ times differentiable, therefore by replacing $m$ with $m-1, g$ with $g^{\Delta}$, and $\alpha=c_{1}$ in (7), we have

$$
g^{\Delta}(p)=\sum_{l=0}^{m-2} v_{l}\left(p, c_{1}\right) g^{\Delta^{l+1}}\left(c_{1}\right)+\int_{c_{1}}^{\rho^{m-2}(p)} v_{m-2}(p, \sigma(\tau)) g^{\Delta^{m}}(\tau) \Delta \tau .
$$

Replace $c_{1}$ with $d_{1}$ in (33) to get

$$
g^{\Delta}(p)=\sum_{l=0}^{m-2} v_{l}\left(p, d_{1}\right) g^{\Delta^{l+1}}\left(d_{1}\right)+\int_{d_{1}}^{\rho^{m-2}(p)} v_{m-2}(p, \sigma(\tau)) g^{\Delta^{m}}(\tau) \Delta \tau .
$$

(4) can be written as

$$
g(p)=\int_{c_{1}}^{d_{1}} z(s) g^{\sigma}(s) \Delta s+\int_{c_{1}}^{p} R_{z}(p, s) g^{\Delta}(s) \Delta s+\int_{p}^{d_{1}} R_{z}(p, s) g^{\Delta}(s) \Delta s .
$$


Now, by using (5), (33), (34), we have

$$
\begin{aligned}
g(p)= & \int_{c_{1}}^{d_{1}} z(s) g^{\sigma}(s) \Delta s+\int_{c_{1}}^{p} Z(s) \sum_{l=0}^{m-2} v_{l}\left(s, c_{1}\right) g^{\Delta^{l+1}}\left(c_{1}\right) \Delta s \\
& +\int_{c_{1}}^{p} Z(s) \int_{c_{1}}^{\rho^{m-2}(s)} v_{m-2}(s, \sigma(\tau)) g^{\Delta^{m}}(\tau) \Delta \tau \Delta s \\
& +\int_{p}^{d_{1}}(Z(s)-1) \sum_{l=0}^{m-2} v_{l}\left(s, d_{1}\right) g^{\Delta^{l+1}}\left(d_{1}\right) \Delta s \\
& +\int_{t}^{d_{1}}(Z(s)-1) \int_{d_{1}}^{\rho^{m-2}(s)} v_{m-2}(s, \sigma(\tau)) g^{\Delta^{m}}(\tau) \Delta \tau \Delta s .
\end{aligned}
$$

By using Lemma 2, we have

$$
\begin{aligned}
& \int_{c_{1}}^{p} Z(s) \sum_{l=0}^{m-2} v_{l}\left(s, c_{1}\right) g^{\Delta^{l+1}}\left(c_{1}\right) \Delta s \\
& \quad=\sum_{l=0}^{m-2} g^{\Delta^{l+1}}\left(c_{1}\right) \int_{c_{1}}^{p} z(s)\left\{v_{l+1}\left(p, c_{1}\right)-v_{l+1}\left(\sigma(s), c_{1}\right)\right\} \Delta s .
\end{aligned}
$$

Similarly

$$
\begin{aligned}
& \int_{p}^{d_{1}}(Z(s)-1) \sum_{l=0}^{m-2} v_{l}\left(s, d_{1}\right) g^{\Delta^{l+1}}\left(d_{1}\right) \Delta s \\
& =\sum_{l=0}^{m-2} g^{\Delta^{l+1}}\left(d_{1}\right) \int_{d_{1}}^{p} z(s)\left\{v_{l+1}\left(\sigma(s), d_{1}\right)-v_{l+1}\left(p, d_{1}\right)\right\} \Delta s .
\end{aligned}
$$

By using Lemma 2 and (9), integral in the 3rd term of (35) becomes

$$
\begin{aligned}
& \int_{c_{1}}^{p} Z(s) \int_{c_{1}}^{\rho^{m-2}(s)} v_{m-2}(s, \sigma(\tau)) g^{\Delta^{m}}(\tau) \Delta \tau \Delta s \\
& \quad=\int_{c_{1}}^{p} g^{\Delta^{m}}(\tau)\left[v_{m-1}(p, \sigma(\tau)) Z(t)-\int_{\rho^{m-3}(\tau)}^{p} Z^{\Delta}(s) v_{m-1}(\sigma(s), \sigma(\tau)) \Delta s\right] \Delta \tau .
\end{aligned}
$$

Similarly,

$$
\begin{gathered}
\int_{p}^{d_{1}}(Z(s)-1) \int_{d_{1}}^{\rho^{m-2}(s)} v_{m-2}(s, \sigma(\tau)) g^{\Delta^{m}}(\tau) \Delta \tau \Delta s \\
=\int_{d_{1}}^{p} g^{\Delta^{m}}(\tau)\left[v_{m-1}(p, \sigma(\tau))(1-Z(p))\right. \\
\left.\quad+\int_{\rho^{m-3}(\tau)}^{p} Z^{\Delta}(s) v_{m-1}(\sigma(s), \sigma(\tau)) \Delta s\right] \Delta \tau .
\end{gathered}
$$

By using (36)-(39) in (35), we have the required result. 
Remark 7 Consider all the assumptions of Theorem 6 hold. Also, assume that $(r, q)$ is a pair of conjugate exponents, that is, $1 \leq r, q \leq \infty, \frac{1}{r}+\frac{1}{q}=1$. Then we have

$$
\begin{aligned}
\mid g(p) & -\int_{c_{1}}^{d_{1}} z(s) g^{\sigma}(s) \Delta s-\sum_{l=0}^{m-2} g^{\Delta^{l+1}}\left(c_{1}\right) \int_{c_{1}}^{p} z(s)\left\{v_{l+1}\left(p, c_{1}\right)-v_{l+1}\left(\sigma(s), c_{1}\right)\right\} \Delta s \\
& -\sum_{l=0}^{m-2} g^{\Delta^{l+1}}\left(d_{1}\right)\left[\int_{d_{1}}^{p} z(s)\left\{v_{l+1}\left(\sigma(s), d_{1}\right)-v_{l+1}\left(p, d_{1}\right)\right\} \Delta s\right] \mid \\
\leq & \left\|g^{\Delta^{m}}\right\|_{r}\left(\int_{c_{1}}^{d_{1}}\left|Q_{z, m}(p, \tau)\right|^{q} \Delta \tau\right)^{\frac{1}{q}} .
\end{aligned}
$$

The constant $\left(\int_{c_{1}}^{d_{1}}\left|Q_{z, m}(p, \tau)\right|^{q} \Delta \tau\right)^{\frac{1}{q}}$ is sharp for $1<r \leq \infty$ and optimal for $r=1$.

Proof This result can be proved by a similar solution used for Theorem 5 .

Remark 8 By using $\mathbb{T}=\mathbb{R}$ in (40), we have $[4,(3.1)]$.

\title{
5 Conclusion
}

In this paper, the extension of Montgomery identity has been obtained with the help of time-scaled Taylor's formula and discussed for calculus (discrete and quantum) as well by choosing special time scales. Further, it is used to find the extension of Ostrowski inequality, mid-point inequality, and trapezoid inequality. The weighted version of Montgomery identity and respective Ostrowski inequality are also established here. Remaining results that appeared in Corollary 1 and in Sect. 3.1 can be proved for weighted Montgomery identity (32) and respective Ostrowski type inequality (40). Moreover, as special cases, our inequalities contain the results proved in $[4]$ when $\mathbb{T}=\mathbb{R}$.

\author{
Acknowledgements \\ The authors wish to thank the editor for consideration of this paper in "Journal of Inequalities and Applications". \\ Funding \\ There is no funding for the research article. \\ Availability of data and materials \\ Information sharing is not appropriate to this paper as no data sets were produced or analyzed during the present study.
}

\section{Declarations}

Competing interests

The authors declare that they have no competing interests.

Authors' contributions

SM wrote the initial draft after calculation of results, KAK originated the idea of this research and supervised the results, the methodology was given by AN, and special cases were confirmed by KMA. All authors read and approved the final manuscript.

\section{Publisher's Note}

Springer Nature remains neutral with regard to jurisdictional claims in published maps and institutional affiliations. 


\section{References}

1. Adil Khan, M., Khan, S., Ullah, I., Ali Khan, K., Chu, Y.M.: A novel approach to the Jensen gap through Taylor's theorem. Math. Methods Appl. Sci. 44(5), 3324-3333 (2021)

2. Akin, E.: Cauchy functions for dynamic equations on a measure chain. J. Math. Anal. Appl. 267(1), 97-115 (2002)

3. Ali, M.A., Chu, Y.M., Budak, H., Akkurt, A., Yaldrim, H., Zahid, M.A.: Quantum variant of Montgomery identity and Ostrowski-type inequalities for the mappings of two variables. Adv. Differ. Equ. 2021(1), 1 (2021)

4. Aljinović, A.A., Pečaríc, J., Vukelíc, A.: On some Ostrowski type inequalities via Montgomery identity and Taylor's formula II. Tamkang J. Math. 36(4), 279-301 (2005)

5. Alshanti, W.G.: Inequality of Ostrowski type for mappings with bounded fourth order partial derivatives. Abstr. Appl. Anal. 2019 (2019)

6. Baleanu, D., Mohammed, P.O., Zeng, S.: Inequalities of trapezoidal type involving generalized fractional integrals. Alex Eng. J. 59(5), 2975-2984 (2020)

7. Bohner, M., Georgiev, S.G.: Multivariable Dynamic Calculus on Time Scales. Springer, Switzerland (2016) https://doi.org/10.1007/978-3-319-47620-9

8. Bohner, M., Matthews, T.: Ostrowski inequalities on time scales. J. Inequal. Pure Appl. Math. 9(1), 8 (2008)

9. Bohner, M., Peterson, A.: Dynamic Equations on Time Scales: An Introduction with Applications. Birkhäuser, Boston (2001)

10. Bohner, M., Peterson, A.: Advances in Dynamic Equations on Time Scales: An Introduction with Applications. Birkhäuser, Boston (2003)

11. Butt, S.I., Khan, K.A., Pečarić, J.: Generalization of Popoviciu inequality for higher order convex function via Tayor's polynomial. Acta Univ. Apulensis, Mat.-Inform. 42, 181-200 (2015)

12. Erden, S., Çelik, N., Khan, M.A.: Refined inequalities of perturbed Ostrowski type for higher-order absolutely continuous functions and applications. AIMS Math. 6(1), 389-404 (2021)

13. Karpuz, B.: Unbounded oscillation of higher-order nonlinear delay dynamic equations of neutral type with oscillating coefficients. Electron. J. Qual. Theory Differ. Equ. 2009, 34 (2009)

14. Khan, K.A., Awan, K.M., Malik, S., Nosheen, A.: Extension of Montgomery identity via Taylor polynomial on time scales. Turk. J. Math. 44(5), 1708-1723 (2020)

15. Khan, M.A., Khurshid, Y., Chu, Y.M.: Hermite-Hadamard type inequalities via the Montgomery identity. Commun. Math Appl. 10(1), 85-97 (2019)

16. Kunt, M., Kashuri, A., Du, T., Baidar, A.W.: Quantum Montgomery identity and quantum estimates of Ostrowski type inequalities. AIMS Math. 5(6), 5439-5457 (2020)

17. Mehmood, N., Agarwal, R.P., Butt, S.I., Pecaric, J.: New generalizations of Popoviciu-type inequalities via new Green's functions and Montgomery identity. J. Inequal. Appl. 2017, 108 (2017)

18. Mitrinovic, D.S., Pečaríc, J.E., Fink, A.M.: Inequalities Involving Functions and Their Integrals and Derivatives, vol. 53. Springer, Berlin (1991)

19. Neang, P., Nonlaopon, K., Tariboon, J., Ntouyas, S.K., Agarwal, P.: Some trapezoid and midpoint type inequalities via fractional (p,q)-calculus. Adv. Differ. Equ. 2021(1), 333 (2021)

20. Pečarić, J.: On the Čebyšev inequality. Bul. Inst. Politehn. Timisoara 25(39), 10-11 (1980)

21. Sarikaya, M.Z., Aktan, N., Yildirim, H.: On weighted Čebyšev-Grüss inequalities on time scales. J. Math. Inequal. 2(2), 185-195 (2008)

22. You, X., Farid, G., Maheen, K.: Fractional Ostrowski type inequalities via generalized Mittag-Leffler function. In: Mathematical Problems in Engineering 2020 (2020)

\section{Submit your manuscript to a SpringerOpen ${ }^{\circ}$ journal and benefit from:}

- Convenient online submission

- Rigorous peer review

- Open access: articles freely available online

- High visibility within the field

- Retaining the copyright to your article

Submit your next manuscript at $>$ springeropen.com 